\title{
Enhanced mass transfer during dwarf nova outbursts by irradiation of the secondary?
}

\author{
Y. Osaki ${ }^{1, \star}$ and F. Meyer ${ }^{2}$ \\ ${ }^{1}$ Department of Astronomy, School of Science, University of Tokyo, Bunkyo-ku, Tokyo 113-0033, Japan \\ e-mail: osaki@astron.s.u-tokyo.ac.jp \\ ${ }^{2}$ Max-Planck-Institut für Astrophysik, Karl-Schwarzschild-Str. 1, 85740 Garching, Germany
}

Received 9 August 2004 / Accepted 9 October 2004

\begin{abstract}
One of the remaining issues in the problems of dwarf novae is whether or not enhanced mass transfer due to irradiation of the secondary stars could occur during outbursts. In a previous paper (Osaki \& Meyer 2003), we presented a theoretical analysis that shows no appreciable enhancement of the mass outflow rate. This conclusion is challenged by Smak (2004) who claims that equations used in our analysis were incorrect and that in systems with short orbital periods substantial enhancement could occur. In this letter, we examine the origin of such divergent conclusions. We show that Smak's solutions are unacceptable from the standpoint of the equation of continuity and that our analysis is an appropriate one to treat this problem.
\end{abstract}

Key words. accretion, accretion disks - binaries: close - novae, cataclysmic variables - stars: dwarf novae

\section{Introduction}

It is well known in theory of outburst mechanisms of dwarf novae that two rival models (i.e., the disk instability model and the mass-transfer burst model) have been competing with each other. The disk instability model is now favored because of substantial evidence both in observations and theory and is widely accepted as the correct mechanism (see, e.g., monographs by Warner 1995; Hellier 2001). However, claims for evidence of enhanced mass overflow due to irradiation of the secondary stars during outbursts of dwarf novae, in particular during the superoutbursts of SU UMa stars, still appear in the literature from time to time. We have critically examined the observational evidence (Osaki \& Meyer 2003) and concluded that it is not well substantiated. Furthermore, we have presented a theoretical analysis which shows that irradiation during outburst should not affect the mass transfer rate. However, this theoretical analysis has recently been challenged by Smak (2004) who claims that our equations were incorrect. The purpose of this letter is to examine critically these two papers and to clarify what the correct approach to treat this problem is.

\section{The problem}

During an outburst of a dwarf nova the surface of the secondary star is irradiated by radiation from the central white dwarf, the inner part of the accretion disk, and the boundary layer, and the temperature of irradiated regions of the secondary star is raised. However, the inner Lagrangian point from which mass

\footnotetext{
* Professor Emeritus, University of Tokyo and Nagasaki University.
}

overflow occurs and the equatorial region of the secondary star are shielded by the accretion disk and only the region of higher latitude is exposed. Unequal irradiation then causes fluid flow over the surface of the secondary. The question is whether this flow is capable to transport heat to the inner Lagrangian point and thereby significantly enhance the mass overflow rate.

Controversy has arisen between Osaki \& Meyer (2003) and Smak (2004) over how to calculate this fluid flow. Osaki \& Meyer (2003) treated it as a steady geostrophic flow using the Eulerian equation of fluid motion while Smak (2004) instead calculates trajectories of fluid particles for a given initial condition using the Lagrangian equation of motion. Although there are several minor differences between the two papers such as treatment of viscosity, coordinate systems used, and temperature of the irradiated part of the surface, they are not essential and thus we concentrate here on the fundamental difference in the treatment of the hydrodynamic flow.

\section{Fluid dynamics in a rotating frame of reference}

The basic equations of fluid flow in a frame of reference rotating with the binary system (see Pedlovsky 1982) are the equation of motion,

$$
\frac{\mathrm{d} \boldsymbol{v}}{\mathrm{d} t}=-\frac{\nabla P}{\rho}-\nabla \Psi-2 \Omega \times \boldsymbol{v}+\frac{\mathcal{F}}{\rho},
$$

and the equation of continuity,

$$
\frac{\mathrm{d} \rho}{\mathrm{d} t}+\rho \nabla \cdot \boldsymbol{v}=0
$$


where $v$ is the flow velocity in the corotating frame, $P$ and $\rho$ are pressure and density, $\Psi$ is the total gravitational ("Roche") potential with contributions from both binary stars and the centrifugal force, $\Omega$ is the angular velocity of rotation of the binary system, $\mathcal{F}$ is the viscous force, and symbols with boldface signify vectors. The Lagrangian time derivative $\frac{\mathrm{d}}{\mathrm{d} t}$ follows a particular fluid element and is related to the Eulerian derivative $\frac{\partial}{\partial t}$ by

$\frac{\mathrm{d}}{\mathrm{d} t}=\frac{\partial}{\partial t}+(\boldsymbol{v} \cdot \nabla)$

The equation of continuity is rewritten in Eulerian form as

$\frac{\partial \rho}{\partial t}+\nabla \cdot(\rho \boldsymbol{v})=0$

In unperturbed state in which irradiation is not yet applied, we assume that the secondary star rotates synchronously and is in hydrostatic equilibrium so that

$\boldsymbol{v}_{0}=0 \quad$ and $\quad \frac{\nabla P_{0}}{\rho_{0}}=-\nabla \Psi$,

where subscript 0 denotes unperturbed equilibrium values.

\section{The two approaches}

We now consider the effect of irradiation. As discussed above, the equatorial zone of the secondary star is shadowed by the accretion disk and only a region of higher latitude on the secondary star is heated by irradiation. The resulting pressure difference between these two regions drives a fluid flow. Since the depth of the zone affected by irradiation (which we denote by $D$ ) is limited to a sub-surface zone a few scale heights deep, we have $D \ll R$, where $R$ is the stellar radius. We may then consider flow in this surface zone only. The flow is essentially horizontal, the ratio of the horizontal to the vertical component of velocity is of the order of $D / R$ as seen from the continuity equation. The treatment of this flow is completely different in Osaki \& Meyer (2003) and in Smak (2004). We examine each of these two approaches.

\subsection{Osaki \& Meyer (2003)}

In Osaki \& Meyer (2003) we assume a steady geostrophic flow over the surface of the secondary star. Since the pressure gradient force caused by uneven heating is directed in latitudinal direction toward the equator and since in geostrophic approximation the Coriolis force balances the pressure gradient, the flow is zonal, i.e., directed parallel to the equator and, for an inviscid fluid, the equator-ward velocity component is zero. Osaki \& Meyer (2003) also considered effects of a possible turbulent viscosity but concluded that the equator-ward flow velocity is so slow that no effective enhancement of the mass overflow rate is expected.

Osaki \& Meyer discussed the particular case of the 2001 outburst of WZ Sge. Basic quantities there are the 80 min orbital period of the binary and a half thickness of $10^{9.4} \mathrm{~cm}$ for the equatorial shadowed belt. When an outburst occurs and the higher latitude region of the secondary star is heated by irradiation, a flow sets in. Its initial phase may be rather complicated but it will settle in an essentially steady state because the typical time-scale of an outburst is of the order of a few days, much longer than the rotation period of the secondary star.

The order of magnitude of the left hand side of Eq. (1) is given by

$\frac{\mathrm{d} \boldsymbol{v}}{\mathrm{d} t}=\frac{\partial \boldsymbol{v}}{\partial t}+(\boldsymbol{v} \cdot \nabla) \boldsymbol{v}=0\left(\frac{V}{\tau}, \frac{V^{2}}{L}\right)$,

where $V, L$, and $\tau$ are characteristic values of velocity, length, and time-scale of the flow. The ratio of the inertial term to the Coriolis force on the right hand side is the so-called Rossby number, Ro,

$\left|\frac{\mathrm{d} v / \mathrm{d} t}{2 \Omega \times v}\right|=0\left(\frac{1}{2 \Omega_{\perp} \tau}, \frac{V}{2 \Omega_{\perp} L}\right)$

where $\Omega_{\perp}$ is the component of the angular velocity perpendicular to the local surface.

In our case the Rossby number given by Ro $=1 /\left(2 \Omega_{\perp} \tau\right)$ is much less than one except for the equatorial region. We may estimate Ro $\sim 0.01$ for the particular case considered by Osaki \& Meyer in which $\Omega_{\perp} \sim 10^{-3.15} \mathrm{~s}^{-1}$ and $\tau \sim 10^{5} \mathrm{~s}$ because we are interested in the flow system on the surface on a timescale of days. In such a case we may neglect the inertial term on the left hand side of the equation of motion with respect to the Coriolis term. The equation of motion for an inviscid fluid then is

$2 \Omega \times v=-\frac{\nabla P}{\rho}-\nabla \Psi$.

Let us use a local Cartesian coordinate system with coordinates $x$ in the direction of constant latitude (eastward), $y$ in the direction of constant longitude (northward), and $z$ normal to the equipotential surface (vertically upward). Since the main gradients are perpendicular to the shadow band, we neglect gradients in the $x$ direction and obtain (Osaki \& Meyer 2003)

$$
\begin{aligned}
-\frac{\partial P}{\partial y}-2 \rho v_{x} \Omega_{\perp} & =0, \\
2 \rho v_{y} \Omega_{\perp} & =0 .
\end{aligned}
$$

Without viscosity we have the "geostrophic" flow along the shadow band

$v_{x}=-\frac{1}{2 \Omega_{\perp}} \frac{1}{\rho} \frac{\partial P}{\partial y} \approx-\frac{1}{2 \Omega_{\perp}} \frac{\Delta c^{2}}{l}$

$v_{y}=0$

where $c=(P / \rho)^{1 / 2}$ is the isothermal sound velocity and $l$ is the half width of the shadow band. Thus the flow velocity is at right angle to the driving pressure gradient which is balanced by the Coriolis force, similar to flows in weather systems and oceans on the rotating earth. Thus without friction no heat can be advected into the shadow region below $L_{1}$. The order of magnitude of the zonal flow generated by the geostrophic balance in Eq. (10) is $V \sim 10^{4} \mathrm{~cm} / \mathrm{s}$ where we used $\Delta c^{2} \sim 10^{10.6} \mathrm{~cm}^{2} / \mathrm{s}^{2}$ and $L \sim 10^{9.4} \mathrm{~cm}$, which yields a corresponding Rossby number Ro $=V /\left(2 \Omega_{\perp} L\right) \sim 0.003$, confirming the self-consistency of the geostrophic flow. 
Here we may add the following notes. As in our previous paper (Osaki \& Meyer 2003), we used above a local Cartesian coordinate system to simplify the complex Roche geometry. However, the detailed geometry is not important in the geostrophic approximation because the geostrophic flow is directed perpendicular to the pressure gradient and as the latter is directed from the shadowed to the irradiated region the flow direction is along the shadow boundary. Flow over the surface of a mass-losing secondary star in a semi-detached binary was already discussed by Lubow \& Shu (1975) who argued that the horizontal flow is parallel to the isobars calling the approximation "astrostrophic" rather than "geostrophic". Our Eq. (10) is exactly the same as their Eq. (66) in their Sect. V. Oka et al (2002) made numerical simulations of the surface flow of a gaslosing secondary star and their numerical simulations basically confirmed Lubow \& Shu's astrostrophic wind.

\subsection{Smak (2004)}

Smak (2004) calculated the motion of fluid elements using the Lagrangian equation of motion. He begins the calculation at the irradiated side of the shadow boundary (which we call "the starting point") and then follows the motion of each fluid element along its trajectory as a one-dimensional initial-value problem. The fundamental objection to this approach is that it does not describe a continuous hydrodynamic flow but only calculates the motion of a free particle that abruptly starts at a particular location following the force of a pressure gradient obtained from cooling of the flow. Although unphysical, let us examine Smak's approach in more detail.

\subsubsection{Quasi-steady case}

Smak (2004) apparently considers a quasi-steady state when in his Eq. (14) he calculates the temperature gradient from its Lagrangian time derivative: the complete Lagrangian derivative of temperature $T$ is

$$
\begin{aligned}
\frac{\mathrm{d} T}{\mathrm{~d} t} & =\frac{\partial T}{\partial t}+\boldsymbol{v} \cdot \nabla T=\frac{\partial T}{\partial t}+v \frac{\mathrm{d} T}{\mathrm{~d} s} \\
& =\frac{\partial T}{\partial t}+v_{x_{*}} \frac{\partial T}{\partial x_{*}}+v_{y_{*}} \frac{\partial T}{\partial y_{*}},
\end{aligned}
$$

where $s$ is the distance from the starting point measured along the trajectory and $x_{*}$ and $y_{*}$ are local Cartesian coordinates used by Smak with $x_{*}$ directed toward the equator and $y_{*}$ directed in longitudinal direction. In Smak's formulation the first and third term of the right hand side are neglected so that

$\frac{\mathrm{d} T}{\mathrm{~d} t}=v_{x_{*}} \frac{\partial T}{\partial x_{*}}$,

i.e., steady state is assumed. Accordingly, in his Fig. 2 the trajectories of fluid elements shown are considered as stream lines of the flow. The third term on the right hand side in Eq. (11) was neglected by Smak because he assumed $\partial T / \partial y_{*}=0$ as his stream lines were fairly parallel.

We therefore adopt the assumption of steady state to further examine Smak's solutions. Taking the scalar product of the equation of motion (Eq. (17) of Smak) with the velocity $v$, we obtain

$\boldsymbol{v} \cdot \frac{\mathrm{d} \boldsymbol{v}}{\mathrm{d} t}=-\frac{\boldsymbol{v} \cdot \nabla P}{\rho}-\boldsymbol{v} \cdot \nabla \Psi$

Using the equation of state $P=(\mathfrak{R} \rho T / \mu)$, with $\mathfrak{R}$ and $\mu$ gas constant and mean molecular weight, respectively, Eq. (13) becomes

$\boldsymbol{v} \cdot \frac{\mathrm{d} v}{\mathrm{~d} t}=-\frac{\mathfrak{R} T}{\mu} \frac{\boldsymbol{v} \cdot \nabla \rho}{\rho}-\frac{\mathfrak{R}}{\mu} \boldsymbol{v} \cdot \nabla T-\boldsymbol{v} \cdot \nabla \Psi$

Smak then assumed that the pressure gradient is mainly produced by the temperature gradient so that a density gradient is neglected and also takes $\boldsymbol{v} \cdot \nabla \Psi=0$ because the flow is confined to the equipotential surface. Then we obtain

$$
\frac{1}{2} \frac{\mathrm{d} v^{2}}{\mathrm{~d} t}=-\frac{\mathfrak{R}}{\mu} \frac{\mathrm{d} T}{\mathrm{~d} t}
$$

In Smak's formulation, the Lagrangian time derivative of temperature on the right hand side of Eq. (15) is given by radiative cooling of the surface element (his Eq. (16)) and he apparently numerically integrated his equations as an initial value problem. However, Eq. (15) can be integrated analytically to yield, along a given stream line,

$\frac{1}{2} v^{2}+\frac{\mathfrak{R} T}{\mu}=$ const.

This corresponds to Bernoulli's equation.

As initial condition Smak apparently assumed that the material at the starting point is at rest, $v=0$ at $s=0$. Otherwise one should not have chosen the starting point at the shadow boundary.

Equation (16) then becomes

$\frac{1}{2} v^{2}+\frac{\mathfrak{R} T}{\mu}=\frac{\mathfrak{R} T_{0}}{\mu}$

where $T_{0}$ is the temperature at the starting point. We note that this relation holds for all the trajectories for a given set of model parameters listed in Smak's Table 2.

At first sight the above solution might look reasonable because gas at rest at the shadow boundary is accelerated to higher velocity by the temperature gradient produced by cooling of the flow. However, this solution has the fatal flaw that it does not satisfy the equation of continuity: if one were to estimate the mass flux from the irradiated to the shadowed side by this formula, he would come to the unbelievable conclusion of no mass flux across the shadow boundary because the velocity there is zero.

One can demonstrate that Smak's solution of Eq. (16) is applicable only as a steady-state solution for supersonic flow. However, as in Smak's solution matter near the shadow boundary is subsonic, it can not be reconciled with the equation of continuity (Eq. (4)) which in steady state is

$\boldsymbol{v} \cdot \nabla \rho+\rho \nabla \cdot \boldsymbol{v}=0$.

A subsonic flow behaves more like an incompressible fluid and the first term on the left hand side of Eq. (18) becomes 
negligible, $\boldsymbol{v} \cdot \nabla \rho=0$, an approximation used by Smak in expressing pressure gradient by temperature gradient in his Eq. (13). However, Smak's accelerating flow has a non-zero velocity divergence in contradiction to the equation of continuity (Eq. (18)). In particular, in his solution the velocity divergence becomes infinite at the shadow boundary from where gas at rest is accelerated to a finite velocity: in Smak's form, Eq. (15) is written as

$v_{x_{*}} \frac{\partial v_{x_{*}}}{\partial x_{*}}=-\frac{\mathfrak{R}}{\mu} \frac{\mathrm{d} T}{\mathrm{~d} t}$.

In the shadow zone the right hand side of Eq. (19) is non-zero so that $\partial v_{x_{*}} / \partial x_{*}$ becomes infinite when $v_{x_{*}}=0$.

This decisive defect of Smak's solution results from his assumption that matter at the shadow boundary stays at rest. But there is no steady flow on parallel equator-ward steamlines with zero velocity at the boundary. If instead gas has a finite velocity across the shadow boundary, matter from higher latitude must move in to replace matter that moved into the shadow region. Since this matter has already a significant zonal velocity when passing the shadow boundary, a picture very different from that of Smak emerges.

One may ask whether at the shadow boundary cool material might well up from below to fill the gap created by gas drained equatorwards, and always replace it by matter with zero zonal velocity. However, in order for up-welling gas to prevent inflow from higher latitude it must provide the same pressure at the same depth as the neighboring irradiated region. With vertical hydrostatic equilibrium this means the same temperature down to the same depth. To heat up a column by irradiation to that depth requires the same time as that for which the existing hot material was exposed i.e. the time since the start of irradiation. As soon as this time exceeds the time on which heated matter flows in, of the order of the sound travel time (about an hour), up-welling of gas is suppressed by inflow.

\subsubsection{Unsteady case}

This shows that Smak's approach is unacceptable as a description of steady flow. Let us examine whether his solutions might reflect an unsteady initial flow. In this case we suddenly switch on the pressure gradient and observe what happens.
As Smak argued, the matter at the heated side of the shadow boundary first moves along the pressure gradient, i.e. equatorwards. This lowers the density at the shadow boundary and creates a pressure deficit with respect to neighboring higher latitude regions from which gas then moves in to fill the void. Since this gas has lower specific angular momentum than that which already left the starting point, it has there a significant zonal velocity. This velocity increases with time as material from higher and higher latitude is drained into the shadow region. This adjustment occurs over all the surface of the secondary star until the flow at the boundary of the shadow region has a zonal component sufficient for the Coriolis force to balance the pressure gradient, and our steady geostrophic state is reached.

One may estimate the time needed to establish geostrophic equilibrium. In the case of WZ Sge discussed above and for the minimal model of a spherical secondary star already gas from a distance of $10^{9.6} \mathrm{~cm}$ from the equator, on reaching the interior of the shadow belt would have enough zonal velocity to balance pressure gradient by Coriolis force. The hydrodynamic time for this matter to arrive in the shadow belt is again only of the order of an hour, the same order of magnitude as the orbital period.

\section{Conclusion}

We conclude that in dwarf novae outbursts enhanced mass transfer by irradiation of the secondary star is prevented by the strong Coriolis force.

\section{References}

Hellier, C. 2001, Cataclysmic Variable Stars: How and Why They Vary (London: Springer)

Lubow, S. H., \& Shu, F. H. 1975, ApJ, 198, 383

Oka, K., Nagae, T., Matsuda, T., Fujiwara, H., \& Boffin, H. M. J. 2002, A\&A, 394, 115

Osaki, Y., \& Meyer, F. 2003, A\&A, 401, 325

Pedlovsky, J. 1982, Geophysical Fluid Dynamics (New York: Springer)

Smak, J. 2004, Acta Astron., 54, 181

Warner, B. 1995, Cataclysmic Variable Stars (Cambridge: Cambridge University Press) 